University of Wollongong

Research Online

Australian Institute for Innovative Materials -

Papers

Australian Institute for Innovative Materials

$1-1-2011$

Molecular-beam epitaxy and robust superconductivity of stoichiometric FeSe crystalline films on bilayer graphene

Canli Song

Tsinghua University, Chinese Academy of Sciences

Yi-Lin Wang

Chinese Academy Of Sciences

Yeping Jiang

Tsinghua University, Chinese Academy of Sciences

Zhi Li

Chinese Academy of Sciences, zhili@uow.edu.au

Lili Wang

Chinese Academy of Sciences

See next page for additional authors

Follow this and additional works at: https://ro.uow.edu.au/aiimpapers

Part of the Engineering Commons, and the Physical Sciences and Mathematics Commons

Research Online is the open access institutional repository for the University of Wollongong. For further information contact the UOW Library: research-pubs@uow.edu.au 


\title{
Molecular-beam epitaxy and robust superconductivity of stoichiometric FeSe crystalline films on bilayer graphene
}

\begin{abstract}
We report on molecular beam epitaxy growth of stoichiometric and superconducting FeSe crystalline thin films on double-layer graphene. Layer-by-layer growth of high-quality films has been achieved in a wellcontrolled manner by using Se-rich condition, which allow us to investigate the thickness-dependent superconductivity of FeSe. In situ low-temperature scanning tunneling spectra reveal that the local superconducting gap in the quasiparticle density of states is visible down to two triple layers for the minimum measurement temperature of $2.2 \mathrm{~K}$, and that the transition temperature Tc scales inversely with film thickness.
\end{abstract}

\section{Keywords}

superconductivity, robust, epitaxy, bilayer, molecular-beam, graphene, stoichiometric, fese, crystalline, films

Disciplines

Engineering | Physical Sciences and Mathematics

\section{Publication Details}

Song, C., Wang, Y., Jiang, Y., Li, Z., Wang, L., He, K., Chen, X., Ma, X. \& Xue, Q. (2011). Molecular-beam epitaxy and robust superconductivity of stoichiometric FeSe crystalline films on bilayer graphene. Physical Review B: Condensed Matter and Materials Physics, 84 020503-1-020503-4.

\section{Authors}

Canli Song, Yi-Lin Wang, Yeping Jiang, Zhi Li, Lili Wang, Ke He, Xi Chen, Xu-Cun Ma, and Qi-Kun Xue 


\title{
$\xi^{\circ}$ \\ Molecular-beam epitaxy and robust superconductivity of stoichiometric FeSe crystalline films on bilayer graphene
}

\author{
Can-Li Song, ${ }^{1,2}$ Yi-Lin Wang, ${ }^{1}$ Ye-Ping Jiang, ${ }^{1,2} \mathrm{Zhi} \mathrm{Li},{ }^{1}$ Lili Wang, ${ }^{1} \mathrm{Ke} \mathrm{He},{ }^{1}$ Xi Chen, ${ }^{2} \mathrm{Xu}-\mathrm{Cun} \mathrm{Ma},{ }^{1,}$ and Qi-Kun Xue ${ }^{1,2, \dagger}$ \\ ${ }^{1}$ State Key Laboratory for Surface Physics, Institute of Physics, Chinese Academy of Sciences, Beijing 100190, China \\ ${ }^{2}$ State Key Laboratory for Low-Dimensional Quantum Physics, Department of Physics, Tsinghua University, Beijing 100084, China
}

(Received 19 May 2011; revised manuscript received 10 June 2011; published 12 July 2011)

\begin{abstract}
We report on molecular beam epitaxy growth of stoichiometric and superconducting FeSe crystalline thin films on double-layer graphene. Layer-by-layer growth of high-quality films has been achieved in a well-controlled manner by using Se-rich condition, which allow us to investigate the thickness-dependent superconductivity of FeSe. In situ low-temperature scanning tunneling spectra reveal that the local superconducting gap in the quasiparticle density of states is visible down to two triple layers for the minimum measurement temperature of $2.2 \mathrm{~K}$, and that the transition temperature $T_{\mathrm{c}}$ scales inversely with film thickness.
\end{abstract}

DOI: 10.1103/PhysRevB.84.020503

PACS number(s): 74.70.Xa, 81.15.Hi, 07.79.Fc, 73.61.At

PbO-type $\beta$-FeSe, with a superconducting transition temperature $T_{\mathrm{c}}$ up to $37 \mathrm{~K}$ under modest pressure, ${ }^{1-3}$ shows some great similarities to the recently discovered high-temperature iron pnictide superconductors, ${ }^{4,5}$ which include the common structural motif of $\mathrm{FeX}_{4}(\mathrm{X}: \mathrm{Se}, \mathrm{As}, \mathrm{P})$ tetrahedra, similar band filling, and low-temperature structural distortion from tetragonal to orthorhombic crystal symmetry. The similarities, together with its simple chemical formula and crystallographic structure, make FeSe an archetype system for unraveling the mechanism of superconductivity in all iron-based superconductors. The FeSe single crystals and films reported so far, however, are known to suffer from great fluctuation in stoichiometry, disorder, and clustering pathologies. ${ }^{1-3,5-7}$ Moreover, for heteroepitaxial FeSe films, the lattice mismatch between the films and substrate introduces compressive or tensile strain into the films, ${ }^{8-12}$ which becomes more dramatic in ultrathin films. These situations make the understanding of their superconductivity more challenging.

In this work, we present a systematic scanning tunneling microscopy (STM) study of molecular beam epitaxy (MBE) growth of FeSe films. ${ }^{13,14}$ Layered and chemically inert double-layer graphene formed on $\mathrm{SiC}(0001)$ was used as substrate. ${ }^{15}$ Since both FeSe and the graphene substrate have a layered structure, intuitively, layer-by-layer growth of unstrained epitaxial films of FeSe should be expected. ${ }^{16-18}$ By optimizing the substrate temperature, we show that stoichiometric and superconducting FeSe films with well-controlled thickness can indeed be prepared under Se-rich condition, and that the stoichiometry is self-regulating, irrespective of the Fe/Se flux ratio. Thickness-dependent superconductivity of the resulting stoichiometric films was then investigated by in situ low-temperature scanning tunneling spectroscopy (STS). It is found that the transition temperature $T_{\mathrm{c}}$ correlates inversely with film thickness $d$, and the thickness for the onset of superconductivity is two triple layers (TL).

Growth of FeSe films was carried out in a standard MBE chamber with a base pressure of $10^{-10}$ torr, and it was connected to a Unisoku low-temperature STM system (down to $2.2 \mathrm{~K}$ ). The double-layer graphene was prepared on a nitrogendoped $\mathrm{SiC}(0001)$ substrate with a resistivity of $\sim 0.1 \Omega \cdot \mathrm{cm}$ using a well-established recipe. ${ }^{15}$ High-purity Fe $(99.995 \%)$ and Se (99.999\%) were used as the source materials. FeSe films were grown by co-evaporating Fe and Se sources from standard Knudsen cells. All STM topographic images were taken at a constant current of $0.1 \mathrm{nA}$ with a polycrystalline PtIr tip. All superconducting gaps were measured using a lock-in technique with a small bias modulation of $0.1 \mathrm{mV}$ at $987.5 \mathrm{~Hz}$, while other STS spectra had a bias modulation of $10 \mathrm{mV}$.

To establish the optimal growth conditions, we started with an analysis of the tetrahedral crystal structure of $\beta$-FeSe, as schematically shown in Fig. 1. Along the (001) crystal direction, ${ }^{1-3}$ one unit cell consists of three atomic layers (one Fe layer and two Se layers), defining a unique TL (Fig. 1). The lattice constant along the c axis is $5.518 \AA$, while it is $3.765 \AA$ in the $a-b$ plane. Within a single TL, iron atoms are covalently coordinated with $2 \mathrm{Se}$ anions above and 2 below the planar iron layer, while the bonding between the adjacent TLs is of weak van der Waals type. Consequently, FeSe films grown on various substrates are usually (001)-oriented and have a Se-terminated surface. ${ }^{8-12}$ Due to the very volatile nature of Se molecules, we attempted to grow FeSe films with a high $\mathrm{Se} / \mathrm{Fe}$ flux ratio of 20 to compensate for the Se losses. A ratio even larger than 20 gives the same result. It turns out that the Se-rich condition could indeed assure Se vacancy-free samples with very high quality. For optimal substrate temperatures $\left(180-480^{\circ} \mathrm{C}\right)$, the stoichiometry of the films is self-regulating: The extra Se molecules cannot be incorporated into the FeSe films, since the substrate temperature is higher than the sublimation temperature of Se. Under these conditions, the growth rate is only linearly dependent on the Fe flux determined by the cell temperature of Fe.

Figures 2(a)-2(d) show typical STM topographic images of the as-grown FeSe films, with a nominal thickness of $\sim 15$ $\mathrm{TL}$, grown at various substrate temperatures. The temperatures of Fe-cell and Se-cell were $1150^{\circ} \mathrm{C}$ and $136^{\circ} \mathrm{C}$, respectively, which leads to a low growth rate of $\sim 0.13 \mathrm{TL} / \mathrm{min}$. At the substrate temperature of $180^{\circ} \mathrm{C}$ [Fig. 2(a)], despite some atomically flat terraces, the films appear discontinuous and contain various thicknesses. On the terraces, triangular islands can be observed, implying hexagonal lattice symmetry of the as-grown films. This is further confirmed in the zoom-in STM image in Fig. 2(e), which was taken on the flat terraces. From 


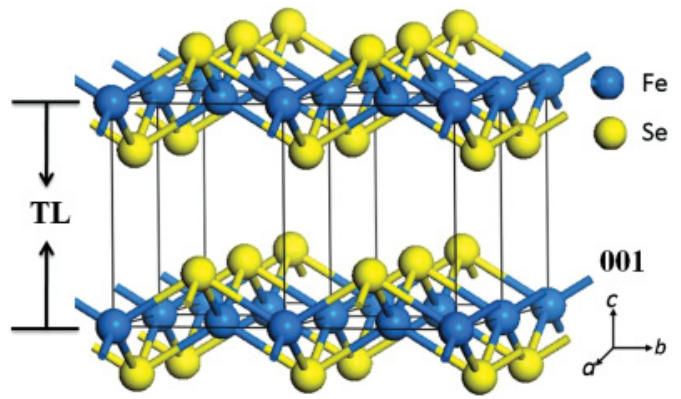

FIG. 1. (Color online) Crystal structure of $\beta$-FeSe.

the high-resolution STM images, the in-plane and out-ofplane lattice constants are measured to be $3.6 \AA$ and $5.7 \AA$, respectively, which agree with the values in the well-known NiAs-type $\alpha$-FeSe. ${ }^{7}$

At elevated substrate temperatures, the films become smoother. This is not surprising because the diffusion of $\mathrm{Fe}$ atoms, which mainly determines the surface morphology in this case, is expected to become stronger at higher temperatures. More importantly, a structural transition from hexagonal to tetragonal or orthorhombic phases was found at a substrate temperature between $180^{\circ} \mathrm{C}$ and $220^{\circ} \mathrm{C}$, as shown in Fig. 2(e) vs Figs. 2(f)-2(h). The lattice parameters of the as-grown films for different substrate temperatures are summarized in Table I. At $220^{\circ} \mathrm{C}$, many bright dumbbell-like pairs occur, and the $1 \times 1$ Se-terminated FeSe surface reconstructs into the well-defined $\sqrt{5} \times \sqrt{5}$ reconstruction with a lattice constant of $\sim 8.5 \AA$ [Fig. 2(f)]. Higher temperature $\left[390^{\circ} \mathrm{C}\right.$; Fig. $\left.2(\mathrm{~g})\right]$ destroys the reconstruction, leaving many dispersed pairs on the surface. All these phases prove to be non-superconductive. Only when the substrate temperature exceeds $420^{\circ} \mathrm{C}$ [for example, $450^{\circ} \mathrm{C}$; Figs. 2(d) and 2(h)] is a square-like lattice observed [see the high-resolution STM image in Fig. 2(h)], and its lattice parameters match well those of bulk $\beta$-FeSe. ${ }^{1}$ Each bright spot in the STM image corresponds to an apical Se atom above the $\mathrm{Fe}$ plane. Low-temperature tunneling spectra exhibit a clear
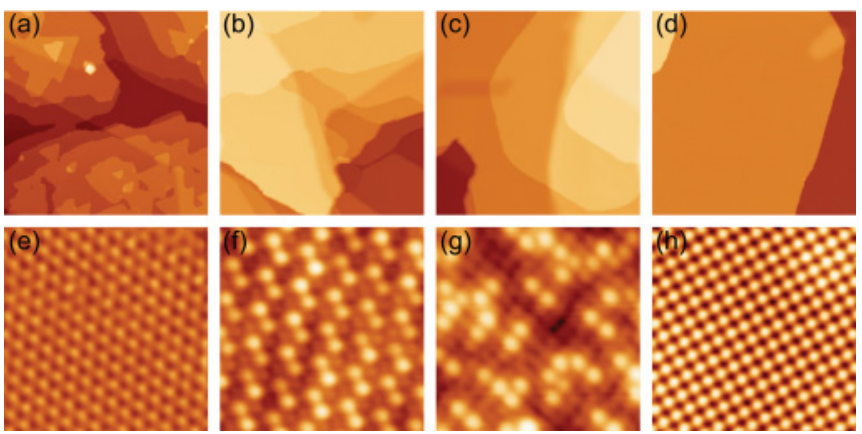

FIG. 2. (Color online) Substrate temperature-dependent film morphology $\left(\mathrm{a}-\mathrm{d}, \mathrm{V}=2.5 \mathrm{~V}, \mathrm{I}=0.1 \mathrm{nA}, 200 \times 200 \mathrm{~nm}^{2}\right)$ and the corresponding atomically resolved STM images $(\mathrm{e}-\mathrm{h}, \mathrm{V}=$ $1 \mathrm{mV}, I=0.1 \mathrm{nA}, 5 \times 5 \mathrm{~nm}^{2}$ ) of the as-grown FeSe films at various substrate temperatures. (a,e) $180^{\circ} \mathrm{C},(\mathrm{b}, \mathrm{f}) 220^{\circ} \mathrm{C},(\mathrm{c}, \mathrm{g}) 390^{\circ} \mathrm{C},(\mathrm{d}, \mathrm{h})$ $450^{\circ} \mathrm{C}$.
TABLE I. The crystal lattice parameters and structure of the asgrown FeSe films at various substrate temperatures. The uncertainty of the lattice constant is smaller than $0.1 \AA$. STM was calibrated on graphene in every measurement.

\begin{tabular}{lcccc}
\hline \hline & \multicolumn{4}{c}{ Temperature $\left({ }^{\circ} \mathrm{C}\right)$} \\
\cline { 2 - 5 } & 180 & 220 & 390 & 450 \\
\hline a $(\AA)$ & 3.6 & 8.5 & 3.8 & 3.8 \\
c $(\AA)$ & 5.7 & 5.5 & 5.5 & 5.5 \\
Structure & hex $^{\text {a }}$ & tet $^{\mathrm{b}}$ or ortho $^{\mathrm{c}}$ & tet $^{\mathrm{b}}$ or ortho $^{\mathrm{c}}$ & $\begin{array}{c}\text { ortho } \\
\text { c }\end{array}$ \\
\hline \hline
\end{tabular}

ahex: hexagonal

b tet: tertragonal

${ }^{c}$ ortho: orthorhombic

superconducting gap near Fermi level $\left(E_{\mathrm{F}}\right)$ with significant spatial homogeneity. ${ }^{19}$

The dumbbell-like pairs in the $\sqrt{5} \times \sqrt{5}$ phase exhibit a strong bias-dependent behavior, suggesting a purely electronic origin of the pairs. At $6 \mathrm{mV}$ [Fig. 3(a)], for example, the STM image shows the aforementioned $\beta$-FeSe (001) lattice, and no pair-like feature is observed. No superconducting signature was found in this surface. Instead, we observed an asymmetric gap of $\sim 0.5 \mathrm{eV}$ near $E_{\mathrm{F}}$ [upper curve in Fig. 3(b)]. Annealing this surface at $450{ }^{\circ} \mathrm{C}$ for a long time $(\sim 4$ hours) can remove the pairs and recover the FeSe (001) surface with a few Se vacancies, as demonstrated in Fig. 3(c). Associated with
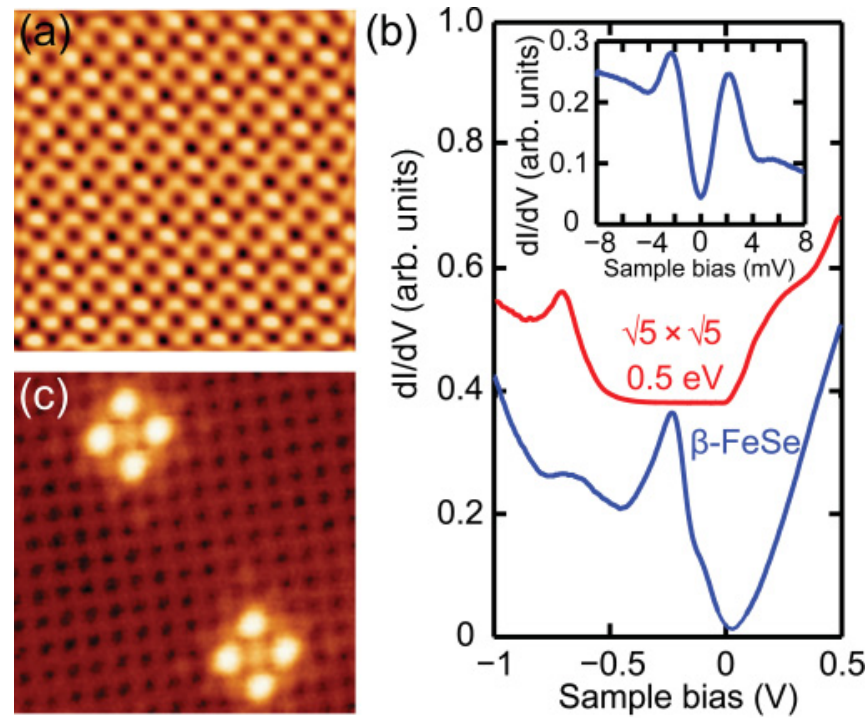

FIG. 3. (Color online) (a) High-resolution STM image $(V=6 \mathrm{mV}$, $I=0.1 \mathrm{nA}, 5 \times 5 \mathrm{~nm}^{2}$ ) taken on the Se-rich $\sqrt{5} \times \sqrt{5}$ surface. The film thickness is $15 \mathrm{TL}$, and the substrate temperature for obtaining the film is $220^{\circ} \mathrm{C}$. (b) $d I / d V$ conductance spectra in the Se-rich (upper curve) and stoichiometric (lower curve) FeSe films, Set point: $V=$ $0.5 \mathrm{~V}, I=0.1 \mathrm{nA}$. The upper curve shifts upward (3.8 a.u.) for clarity. Inset: Low-energy $d I / d V$ spectrum showing the superconducting gap in the stoichiometric $8 \mathrm{TL}$ FeSe film measured at $3.0 \mathrm{~K}$. Set point: $V=10 \mathrm{mV}, I=0.1 \mathrm{nA}$. (c) High-resolution STM image after annealing the Se-rich $\sqrt{5} \times \sqrt{5}$ surface (like in part (a) at $450^{\circ} \mathrm{C}(V=-1 \mathrm{mV}$, $I=0.1 \mathrm{nA}, 5 \times 5 \mathrm{~nm}^{2}$ ). Two Se vacancies are observed. 

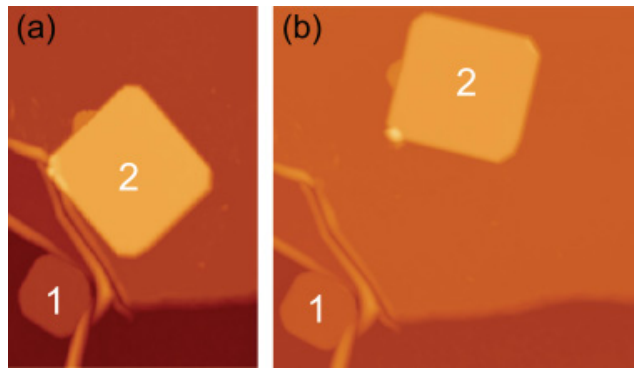

FIG. 4. (Color online) (a,b) Consecutive STM images $(V=2.5 \mathrm{~V}$, $I=0.1 \mathrm{nA}$ ) showing the displacement and rotation of an FeSe island. Image size: (a) $145 \times 100 \mathrm{~nm}^{2}$, (b) $145 \times 145 \mathrm{~nm}^{2}$.

the structural transition, the films become metallic [lower curve in Fig. 3(b)]. Careful STS measurement reveals a superconducting gap near $E_{\mathrm{F}}$ again, as shown in the inset of Fig. 3(b). Based on these observations, we believe that the pairs correspond most likely to excess Se because it is rather difficult for $\mathrm{Fe}$ to evaporate at $450^{\circ} \mathrm{C}$. This is further confirmed by the following experiment: When Se molecules were deposited onto a $\beta$-FeSe $(001)$ surface at $220^{\circ} \mathrm{C}$, the same pairs appear, increase in number and finally evolve into the $\sqrt{5} \times \sqrt{5}$ phase.

Our study reveals that the superconducting gap exists only within a small window $(2.5 \%)$ of extra Se atoms, ${ }^{19}$ and that the superconductivity is very sensitive to the stoichiometry of FeSe. This may explain the controversial experimental results of iron-based superconductors in previous studies, where unavoidable stoichiometry fluctuation occurs in the samples. In our case, however, by using the Se-rich condition and well-controlled substrate temperature, stoichiometric $\beta$-FeSe films with extremely few defects $(\sim 1$ defect per $70000 \mathrm{Se}$ atoms) can be easily prepared. ${ }^{19}$

The advantage of using graphene as a substrate is illustrated in Figs. 4(a)-4(b). By continuously imaging small FeSe islands on graphene, one can clearly observe the scanning-induced displacement and rotation of an FeSe island with a size of $50 \times 50 \mathrm{~nm}^{2}$. This means that the interaction of the FeSe film with the underlying substrate is rather weak,$^{20}$ and FeSe nearly "floats" on the double-layer graphene. Atomic resolution STM images of ultrathin FeSe films (1-4 TL $)^{19}$ reveal that they have the same lattice constant as thick films and bulk $\beta$-FeSe, suggesting a fully relaxed FeSe film even at the first TL.

Figure 5(a) shows a series of normalized tunneling spectra taken on $8 \mathrm{TL}$ FeSe film at various temperatures, which were obtained by dividing each spectrum by the normal-state conductance data just above $T_{\mathrm{c}}$, i.e., $10 \mathrm{~K}$ for $8 \mathrm{TL}$. At $3.0 \mathrm{~K}$, the superconducting gap with two symmetric coherence peaks at $\sim \pm 2.1 \mathrm{meV}$ is clearly visible. With increasing temperature, both coherence peaks are suppressed, and the zero bias conductance (ZBC) continuously increases until the gap completely disappears at $8.0 \mathrm{~K}$. Using the tunneling spectra near $T_{\mathrm{c}}$, ZBC shows a linear dependence on temperature [the inset of Fig. 5(a)]. By extrapolating $T_{\mathrm{c}}$ to the point where $\mathrm{ZBC}=1$, we find a $T_{\mathrm{c}}$ of $7.8 \mathrm{~K}$ for $8 \mathrm{TL}$ film. Similarly, we can determine the $T_{\mathrm{c}}$ of other films, for example, $3.7 \mathrm{~K}$ for $2 \mathrm{TL}$ film [Fig. 5(b)].
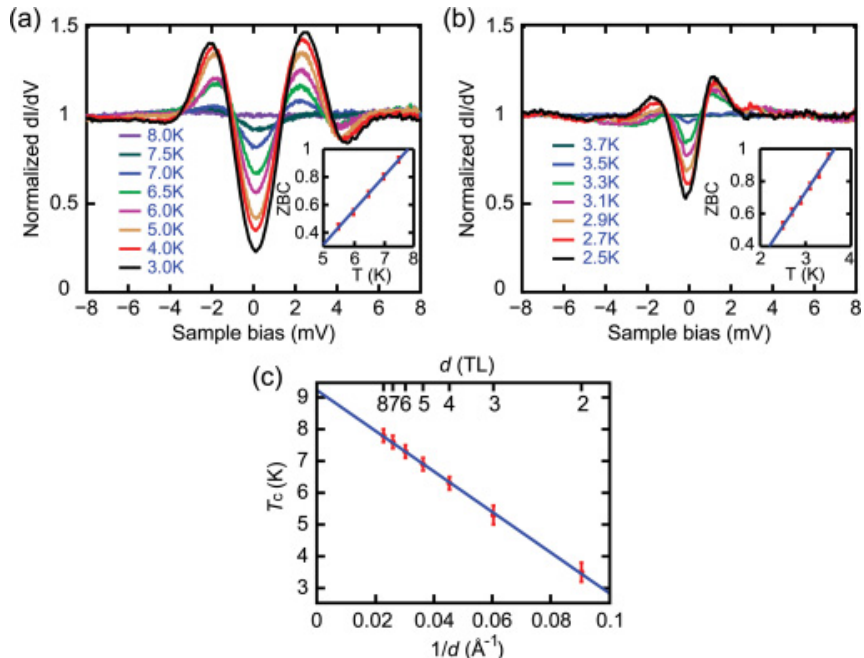

FIG. 5. (Color online) (a,c) A series of normalized tunneling conductance spectra on (a) $8 \mathrm{TL}$ and (b) 2 TL FeSe films. Insets: Temperature-dependent zero bias conductance (ZBC) for (a) $8 \mathrm{TL}$ and (b) $2 \mathrm{TL}$ FeSe films. The bias modulation is set at $0.1 \mathrm{mV}$. (c) Superconducting transition temperature $T_{\mathrm{c}}$ vs the inverse of the film thickness $d$.

In $1 \mathrm{TL}$ film, however, the observed gap near $E_{\mathrm{F}}$ is temperatureindependent, and it exhibits significant spatial inhomogeneity. This suggests that $1 \mathrm{TL}$ FeSe film is non-superconductive above $2.2 \mathrm{~K}$.

Figure 5(c) shows the relationship between $T_{\mathrm{c}}$ and the inverse of film thickness $1 / d$. $T_{\mathrm{c}}$ values scale inversely with the film thickness $d$. As shown previously, for superconducting films such as $\mathrm{Pb}$ and $\mathrm{YBa}_{2} \mathrm{Cu}_{3} \mathrm{O}_{\mathrm{y}},{ }^{21,22}$ the transition temperature $T_{\mathrm{c}}(d)$ is scaled as $T_{\mathrm{c}}(d)=T_{\mathrm{c} 0}\left(1-d_{\mathrm{c}} / d\right)$, where $T_{\mathrm{c} 0}$ $(d=\infty)$ is the critical temperature of the bulk, and $d_{\mathrm{c}}$ is the threshold for the onset of superconductivity. Theoretically, $1 / d$ dependence of $T_{\mathrm{c}}$ has generally been interpreted by adding a surface-energy term in the Ginzburg-Landau freeenergy of a superconductor. ${ }^{23}$ Based on this relation, $d_{c}$ is estimated at $7 \AA$. In terms of the out-of-plane lattice constant of $5.518 \AA$ for $\beta$-FeSe, this suggests that the minimum thickness for superconducting FeSe films is 2 unit cells, consistent with our experiment. Meanwhile, the extrapolation to infinite thickness $(1 / d=0)$ yields a $T_{\mathrm{c} 0} \approx 9.3 \mathrm{~K}$. This agrees well with the bulk $T_{\mathrm{c}}$ of the stoichiometric $\mathrm{FeSe}$ samples. ${ }^{24}$

In summary, we have successfully prepared stoichiometric and superconducting FeSe films on graphene by using MBE. We show that the graphene grown on $\mathrm{SiC}(0001)$ substrate can lead to growth of strain-free FeSe films. By STM/STS, we show that the superconductivity transition temperature $T_{\mathrm{c}}$ of FeSe films $(\geqslant 2 \mathrm{TL})$ scales inversely with the thickness. The growth conditions established here may prove useful for growing other iron-based superconductor films with high quality.

This work was supported by National Science Foundation and Ministry of Science and Technology of China. All STM topographic images were processed by WSxM software (www.nanotec.es). 
*xcma@aphy.iphy.ac.cn

${ }^{\dagger}$ qkxue@mail.tsinghua.edu.cn

${ }^{1}$ F. C. Hsu, J. Y. Luo, K. W. Yeh, T. K. Chen, T. W. Huang, P. M. Wu, Y.-C. Lee, Y. L. Huang, Y. Y. Chu, D. C. Yan, and M. K. Wu, Proc. Natl. Acad. Sci. USA 105, 14262 (2008).

${ }^{2}$ Y. Mizuguchi, F. Tomioka, S. Tsuda, T. Yamaguchi, and Y. Takano, Appl. Phys. Lett. 93, 152505 (2008).

${ }^{3}$ S. Medvedev, T. M. McQueen, I. A. Troyan, T. Palasyuk, M. I. Eremets, R. J. Cava, S. Naghavi, F. Casper, V. Ksenofontov, G. Wortmann, and C. Felser, Nat. Mater. 8, 630 (2009).

${ }^{4}$ J. Paglione, and R. L. Greene, Nat. Phys. 6, 645 (2010) and references therein.

${ }^{5}$ D. C. Johnston, Adv. Phys. 59, 803 (2010).

${ }^{6}$ M. K. Wu, F. C. Hsu, K. W. Yeh, T. W. Huang, J. Y. Luo, M. J. Wang, H. H. Chang, T. K. Chen, S. M. Rao, B. H. Mok, C. L. Chen, Y. L. Huang, C. T. Ke, P. M. Wu, A. M. Chang, C. T. Wu, and T. P. Perng, Physica C 469, 340 (2009).

${ }^{7}$ T. M. McQueen, Q. Huang, V. Ksenofontov, C. Felser, Q. Xu, H. Zandbergen, Y. S. Hor, J. Allred, A. J. Williams, D. Qu, J. Checkelsky, N. P. Ong, and R. J. Cava, Phys. Rev. B 79, 014522 (2009).

${ }^{8}$ Y. Han, W. Y. Li, L. X. Cao, S. Zhang, B. Xu, and B. R. Zhao, J. Phys. Condens. Matter 21, 235702 (2009).

${ }^{9}$ Y. F. Nie, E. Brahimi, J. I. Budnick, W. A. Hines, M. Jain, and B. O. Wells, Appl. Rev. Lett. 94, 242505 (2009).

${ }^{10}$ M. J. Wang, J. Y. Luo, T. W. Huang, H. H. Chang, T. K. Chen, F. C. Hsu, C. T. Wu, P. M. Wu, A. M. Chang, and M. K. Wu, Phys. Rev. Lett. 103, 117002 (2009).
${ }^{11}$ S. X. Huang, C. L. Chien, V. Thampy, and C. Broholm, Phys. Rev. Lett. 104, 217002 (2010).

${ }^{12}$ M. Jourdan, and S. Haaf, J. Appl. Phys. 108, 023913 (2010).

${ }^{13}$ T. Kawaguchi, H. Uemura, T. Ohno, M. Tabuchi, T. Ujihara, K. Takenaka, Y. Takeda, and H. Ikuta, Appl. Rev. Lett. 97, 042509 (2010).

${ }^{14}$ S. Agatsuma, T. Yamagishi, S. Takeda, and M. Naito, Physica C 470, 1468, (2010).

${ }^{15}$ J. Hass, W. A. D. Heer, and E. H. Conrad, J. Phys. Condens. Matter 20, 323202 (2008).

${ }^{16}$ Koma, K. Sunouchi, and T. Miyajima, J. Vac. Sci. Technol. 3, 724 (1985).

${ }^{17}$ Koma, and K. Yoshimura, Surf. Sci. 174, 556 (1986).

${ }^{18}$ K. Ueno, K. Saiki, T. Shimada, and A. Koma, J. Vac. Sci. Technol. A 8, 68 (1990).

${ }^{19}$ C. L. Song, Y. L. Wang, P. Cheng, Y. P. Jiang, W. Li, T. Zhang, Z. Li, K. He, L. L. Wang, J. F. Jia, H. H. Hung, C. J. Wu, X. C. Ma, X. Chen, and Q. K. Xue, Science 332, 1410 (2011).

${ }^{20}$ W. D. Xiao, S. S. Kushvaha, and X. S. Wang, J. Phys. Condens. Matter 20, 225002 (2008).

${ }^{21}$ M. M. Özer, R. J. R. Thompson, and H. H. Weitering, Nat. Phys. 2, 173 (2006).

${ }^{22}$ W. H. Tang, C. Y. Ng, C. Y. Yau, and J. Gao, Supercond. Sci. Technol. 13, 580 (2000)

${ }^{23}$ J. Simonin, Phys. Rev. B 33, 7830 (1986).

${ }^{24}$ Y. J. Song, J. B. Hong, B. H. Min, and K. Jun [http://arxiv.org/abs/0911.2045] (2009). 